\title{
High-risk type-2 diabetes mellitus patients, without prior ischemic events, have normal blood platelet functionality profiles: a cross-sectional study
}

\author{
Gadi Shlomai ${ }^{1,2,3^{* \dagger}}$, Tal Haran-Appe ${ }^{3 \dagger}$, Tal Sella ${ }^{4}$, Yoni Grossman ${ }^{1}$, Hagit Hauschner ${ }^{5}$, Nurit Rosenberg ${ }^{3,5}$
} and Ehud Grossman ${ }^{1,3}$

\begin{abstract}
Background: Patients with type 2 diabetes mellitus (DM) display a predisposition for vascular disease. Platelets taken from vasculopathic diabetic patients, show enhanced stimuli-induced activation and aggregation responses. Aspirin remains the cornerstone antiplatelet agent for secondary prevention of vascular complications among diabetic patients, yet evidence of its efficacy and safety in primary prevention are conflicting. Our aim was to assess whether high risk diabetic patients, without previous ischemic events, have abnormal platelet functionality profiles.

Methods: The study included 82 diabetic patients and 86 matched non-diabetic patients without prior ischemic events nor treatment with anti-platelet medications. Blood samples were analyzed for platelet markers of activation, turnover and leukocyte-platelet interactions.

Results: Our final analysis included 122 males (74\%), with a mean age of 61 years. Mean platelet volume (MPV) was similar between the diabetic patients and controls ( $9.2 \mathrm{fL}$ for both). Following activation, PAC-1 binding and P-selectin expression were found comparable between the diabetic patients and controls ( $83 \%$ versus $81 \%$ and $76 \%$ versus $74 \%$, respectively). Leukocyte-platelet aggregates (LPAs) were similar between the diabetic patients and controls (18\% versus $17 \%$, respectively). Neutrophil-platelet aggregates (NPAs) and monocyte-platelet aggregates (MPAs) were also found similar in the diabetic patients and controls. Elevated fasting plasma glucose was associated with increased LPAs rates.
\end{abstract}

Conclusions: High risk type-2 diabetes mellitus patients, without prior ischemic events, have normal blood platelet functionality profiles.

Keywords: Diabetes mellitus, Platelets activity, Primary prevention, Cardiovascular risk, Aspirin

\section{Background}

Patients with type 2 diabetes mellitus (DM) display a predisposition for accelerated atherosclerosis, manifested as a $2-4$ fold increased risk for premature cardiovascular (CV) disease [1]. Blood platelets play a pivotal role in the blood clotting process by mediating the primary phase of hemostasis. Their involvement in atherogenesis and thrombotic complications has been previously well

\footnotetext{
*Correspondence: Gadi.Shlomai@sheba.health.gov.il

${ }^{\dagger}$ Equal contributors

'Department of Internal Medicine D and Hypertension Unit, The Chaim Sheba Medical Center, Derech Sheba 1, Tel Hashomer, Ramat-Gan 52621, Israel

${ }^{2}$ The Dr. Pinchas Borenstein Talpiot Medical Leadership Program 2013, Tel-Aviv, Israel

Full list of author information is available at the end of the article
}

documented [2,3]. Platelets taken from diabetic patients, particularly those with vascular instability and angiopathy, have been found to have increased baseline activation levels as well as enhanced stimuli-induced activation and aggregation responses (platelet hyper-reactivity) [4]. The pathophysiology underlying the dysregulated signaling pathways of platelets in DM is multifactorial and associated with the pernicious effects of persistent hyperglycemia [5-10], an abnormal lipid profile [11-13], insulin resistance [14-16] and oxidative stress [17, 18].

Diabetic patients present with persistent thromboxanedependent platelet activation, with aspirin playing a pivotal role in the prevention of vascular complications in DM [19]. Nonetheless, while aspirin remains the cornerstone 
anti- platelet agent for secondary prevention of vascular complications among all patients, irrespective of DM status [20], direct evidence of its efficacy and safety in primary prevention is lacking or at best inconclusive [21-24].

In order to resolve discrepancies among the results obtained in different clinical trials, several meta-analyses were conducted. However, these studies demonstrated that primary prevention with aspirin generates only a modest beneficiary effect on CV risk reduction [25-29]. Recently, the American Diabetes Association (ADA) published guidelines stating that prophylactic aspirin therapy appears to have a modest effect on ischemic vascular events with an absolute decrease in events, depending on the underlying CV risk [30].

The 2014 European Society of Cardiology (ESC)/ European Association for the Study of Diabetes (EASD) guidelines also do not recommend primary prevention with aspirin for low risk patients [31]. Nevertheless, the ADA recommended that primary prophylaxis with aspirin therapy for diabetic patients is indicated for those who are at an increased cardiovascular risk (10-year risk $>10 \%$ ), which includes most men aged $>50$ years or women aged $>60$ years who have at least one additional major risk factor [30]. These recommendations essentially support aspirin primary prophylaxis for the majority of diabetic patients.

Real-life data regarding platelet activity and reactivity in high $\mathrm{CV}$ risk diabetic patients without previous $\mathrm{CV}$ events, are limited and conflicting. Herein, we evaluate whether high risk diabetic patients without previous ischemic events and not treated with anti-platelet medications, have abnormal platelet functionality profiles.

\section{Methods}

\section{Subjects}

Subjects included were examined at the Institute for Medical Screening, Chaim Sheba Medical Center, Israel between June 2014 and January 2015. Out of 2337 scheduled subjects, we identified 235 diabetic patients and 2102 non-diabetics patients. Patients were excluded if they had had a past medical history of $\mathrm{CV}$ disease or had been treated with any anti-platelet medications or anticoagulants. Pregnant women were also excluded. Out of 235 diabetic patients, 107 were excluded and 46 refused consent or did not appear for their scheduled annual examination. Out of 2102 non-diabetic patients, 1546 were excluded and 570 patients refused consent, did not appear for their scheduled annual examination or did not match the diabetic patients. Subsequently, the study group included 82 diabetic patients and 86 nondiabetic subjects matched for age, gender, body mass index (BMI), co morbidities, smoking status, medications and renal functions (Fig. 1).
The study protocol was approved by the local institutional review board and complies with the Helsinki declaration. Written consent was given by all subjects.

\section{Definitions}

Diabetes mellitus was defined when either a diagnosis of DM was documented in the medical chart; when fasting plasma glucose was $>126 \mathrm{mg} / \mathrm{dL}(7.0 \mathrm{mmol} / \mathrm{L})$ on two separate readings; the hemoglobin A1C (HbA1C) level was $\geq 6.5 \%(47.5 \mathrm{mmol} / \mathrm{mol})$ or when the subject had taken insulin or oral hypoglycemic medications.

Hypertension was defined when either two separate blood pressure (BP) readings were $\geq 140 \mathrm{mmHg}$ for systolic BP and/or $\geq 90 \mathrm{mmHg}$ for diastolic BP; a history of hypertension was reported or when the subject had taken antihypertensive medications. Hyperlipidemia was defined when the diagnosis was documented in the medical chart or when the patient had been chronically prescribed statins or fibrates. Smoking status was determined according to a questionnaire where participants were divided into current or non-smokers. BMI was calculated from the height and weight parameters measured on the day of inclusion.

Information regarding prescribed medications was obtained from documented the medical charts. All information regarding clinical diagnoses and medical therapy was cross referenced and confirmed with the patients upon signing of the informed consent form.

\section{Protocol}

Upon consent, venous blood was drawn for a complete blood count, renal function tests, serum electrolytes, serum fasting glucose, HbA1C level, a fasting lipid profile, mean platelet volume (MPV) and platelet functionality assays.

$\mathrm{CV}$ risk assessment was conducted according to the "General Cardiovascular Risk Profile of the Framingham Heart Study" [32]. This cardiovascular risk score calculator was utilized for its inclusion of diabetic patients.

\section{Platelet functionality assays}

Venous blood was drawn into a $3.2 \%$ sodium citrate blood-collecting tube. Whole blood was diluted fivefold by adding a $\mathrm{Ca}^{2+}$-free HEPES buffer $(145 \mathrm{mM} \mathrm{NaCl}$, $5 \mathrm{mM} \mathrm{KCl}, 1 \mathrm{mM} \mathrm{MgSO} 4,0.5 \mathrm{mM} \mathrm{NaH2PO} 4,5 \mathrm{mM}$ glucose, and10mM Hepes/Na; Sigma) within $30 \mathrm{~min}$ of collection. Diluted blood samples were incubated for $10 \mathrm{~min}$ at room temperature with PC5-conjugated antiCD45 mAb (BD Bioscience, San Jose, CA) and FITCconjugated anti-CD41 mAb (Beckman Coulter, Marseille, France) for leukocyte-platelet aggregate analysis. Next, samples were fixed with $1 \%$ paraformaldehyde (Electron Microscopy Sciences, Hatfield, PA) for $10 \mathrm{~min}$ at room temperature, followed by a dilution of 4.6-fold with 


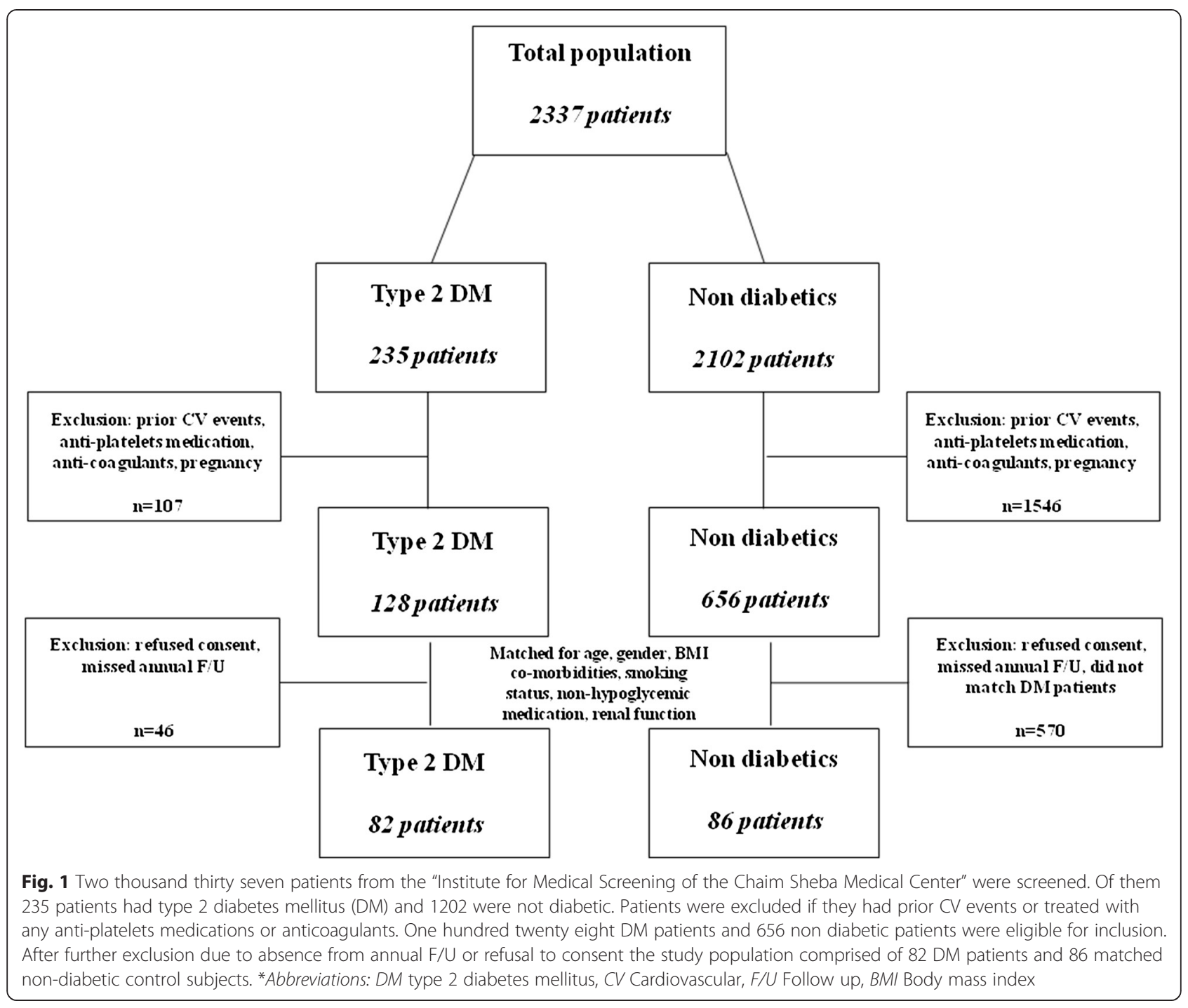

distilled water for red cell lysis and sample dilution. Samples were analyzed by flow cytometry [33]. For detection of platelet activation, blood samples were centrifuged for $10 \mathrm{~min}$ at $800 \mathrm{rpm}$ in preparation for platelet rich plasma (PRP). The PRP were diluted tenfold with PBS and incubated for $20 \mathrm{~min}$ at room temperature with an anti PEconjugated anti CD62p monoclonal antibody (BioLegend, San-Diago, CA) and a FITC- conjugated PAC-1 monoclonal antibody (Becton Dickinson. San Jose, CA), specific to the active conformation of integrin $\alpha \operatorname{IIb} \beta 3$ in the presence or absence of $10 \mu \mathrm{M}$ ADP (molab) as a platelet activator. Samples were diluted fivefold with PBS and analyzed by flow cytometry.

\section{Statistical analysis}

For the univariate analysis, percentages were calculated for categorical variables and means with standard deviations, for continuous variables. The chi-square test for categorical variables and unpaired $t$-test for continuous variables were performed to determine significant differences in baseline characteristics and platelet functions between diabetic patients and controls. Multiple linear regression was performed to assess predictors of platelet function.

Models were adjusted for age, gender, $\mathrm{HbA1C}$, fasting plasma glucose (FPG), low density lipoprotein (LDL), high density lipoprotein (HDL), total cholesterol, hemoglobin, platelet count, estimated glomerular filtration rate (eGFR) and Framingham risk score (FRS). All p value calculations were two-tailed and considered statistically significant if their value was $<0.05$. Statistical analyses were performed by the IBM SPSS version 20.0 (Chicago, Illinois, USA).

\section{Results}

\section{Participants' characteristics}

The study included 82 diabetic patients (74\% males) with a mean age of $62 \pm 8$ years. The controls $(n=86)$ were matched for age, gender, BMI, smoking status, 
renal function, CV related co-morbidities and nonhypoglycemic medications (Table 1). Compared to the controls, diabetic patients had higher fasting plasma glucose levels $(p<0.001)$, elevated HbA1C levels $(p<0.001)$, a higher CV risk as represented by FRS $(p<0.001)$, and lower HDL $(p=0.04)$, LDL $(p<0.001)$ and total cholesterol levels $(p<0.001)$ (Table 1$)$.

\section{Platelet functionality assays}

MPVs were similar between diabetic patients and controls $(9.2 \pm 1.3 \mathrm{fL}$ for both, $p=0.9)$.

Table 1 Basic characteristics

\begin{tabular}{|c|c|c|c|}
\hline Characteristic & Cases (DM) & Controls & $P$ value \\
\hline$n$ & 82 & 86 & \\
\hline Gender (male) - n (\%) & $61(74)$ & $61(74)$ & 0.615 \\
\hline Age (y) - mean (SD) & $62( \pm 8)$ & $62( \pm 7)$ & 0.941 \\
\hline $\mathrm{BMI}(\mathrm{kg} / \mathrm{m} 2)-$ mean $(\mathrm{SD})$ & $28( \pm 4)$ & $27( \pm 4)$ & 0.12 \\
\hline Active smoking $-n(\%)$ & $4(5)$ & $6(7)$ & 0.747 \\
\hline FRS - mean (SD) & $26( \pm 13)$ & $16( \pm 9)$ & $<0.001$ \\
\hline \multicolumn{4}{|l|}{ Comorbidities - n (\%) } \\
\hline Hypertension & $45(55)$ & $47(55)$ & 0.976 \\
\hline Hyperlipidemia & $61(74)$ & $62(72)$ & 0.737 \\
\hline \multicolumn{4}{|c|}{ Hypoglycemic medications - $n$ (\%) } \\
\hline Biguanides & $58(70)$ & $\mathrm{N} / \mathrm{A}$ & \\
\hline GLP1-RA & $3(4)$ & $\mathrm{N} / \mathrm{A}$ & \\
\hline DPP-4 antagonists & $25(30)$ & $\mathrm{N} / \mathrm{A}$ & \\
\hline SU/Meglitinides & $10(12)$ & $\mathrm{N} / \mathrm{A}$ & \\
\hline Insulin & $8(10)$ & $\mathrm{N} / \mathrm{A}$ & \\
\hline \multicolumn{4}{|l|}{ Medications - $n(\%)$} \\
\hline Beta blockers & $16(20)$ & $12(14)$ & 0.334 \\
\hline Calcium channel blockers & $14(17)$ & $17(20)$ & 0.653 \\
\hline ACEi/ARB & $33(40)$ & $31(36)$ & 0.575 \\
\hline Diuretics & $4(5)$ & $9(10)$ & 0.175 \\
\hline Statins & $53(65)$ & $46(54)$ & 0.142 \\
\hline \multicolumn{4}{|l|}{ Laboratory values - mean (SD) } \\
\hline Hemoglobin (g/dl) & $14( \pm 1)$ & $14( \pm 1)$ & 0.635 \\
\hline Platelets (K/microL) & $222( \pm 54)$ & $216( \pm 52)$ & 0.401 \\
\hline $\begin{array}{l}\text { Fasting plasma } \\
\text { glucose (mg/dl) }\end{array}$ & $136( \pm 29)$ & $91( \pm 8)$ & $<0.001$ \\
\hline HbA1c (\%) (mmol/mol) & $6.7( \pm 0.9)(49.7)$ & $5.4( \pm 0.3)(35.5)$ & $<0.001$ \\
\hline $\mathrm{LDL}(\mathrm{mg} / \mathrm{dl})$ & $109( \pm 24)$ & $121( \pm 24)$ & $<0.001$ \\
\hline $\mathrm{HDL}(\mathrm{mg} / \mathrm{dl})$ & $46( \pm 14)$ & $50( \pm 12)$ & 0.040 \\
\hline Total Cholesterol (mg/dl) & $162( \pm 30)$ & $179( \pm 31)$ & $<0.001$ \\
\hline eGFR (mL/min/1.73 m²) & $79( \pm 19)$ & $76( \pm 15)$ & 0.289 \\
\hline
\end{tabular}

DM type 2 diabetes mellitus, BMI Body Mass Index, FRS Framingham Risk Score, GLP-1RA Glucagon Like Peptide-1 Receptor Agonist, DPP-4 Dipeptidyl Peptidase-4, SU Sulfanilurea; ACEi Angiotensin Converting Enzyme Inhibitor, $A R B$ Angiotensin Receptor Blockers, HbA1C Hemoglobin A1C, LDL Low Density Lipoprotein, HDL High Density Lipoprotein, eGFR Estimated Glomerular Filtration Rate
Following ADP activation, the percentage of cells positive for PAC-1 binding and P-selectin expression, markers for fibrinogen receptor activity and $\alpha$-granule secretion, respectively, were comparable between diabetic patients and controls $(83 \%$ for diabetic patients versus $81 \%$ for controls, $p=0.17$, and $76 \%$ for diabetic patients versus $74 \%$ for controls, $p=0.14$, respectively) (Fig. 2). Similarly, calculating the mean fluorescence intensity (MFI) following ADP activation also demonstrated comparable results between groups (an 11.9- fold increase in diabetic patients versus an 11.6- fold increase in non-diabetic patients and a 13.3- fold increase for diabetic patients versus a 13.8fold increase in non-diabetic patients, respectively, data not shown). Moreover, the MFI of PAC-1 and P-selectin for resting platelets was also similar $(10.67 \pm 10.99$ in diabetic patients versus $9.09 \pm 8.2$ in non-diabetic patients and $11.38 \pm 8.98$ in diabetic patients versus $9.86 \pm 7.4$ in non-diabetic patients, respectively, data not shown). These results indicate that both the baseline platelet activation and the ADP-induced activation response were similar for both diabetic patients and controls.

A similar total of leukocyte-platelet aggregates (LPAs) in diabetic patients and non-diabetic patients (18\% versus $17 \%$, respectively, $p=0.25$ ) were found in the leukocyte aggregation assays. Neutrophil-platelet aggregates (NPAs) and monocyte-platelet aggregates (MPAs) were also found to be similar (14 \% in diabetic patients versus $15 \%$ in non-diabetic patients, $p=0.68$, and $22 \%$ in diabetic patients versus $21 \%$ in non-diabetic patients, $p=0.36$, respectively) (Fig. 3).

\section{Effects of glycemic burden and cardiovascular risk on platelets functionality}

The multivariate linear regression model demonstrated that elevated fasting plasma glucose was associated with increased LPAs $(p=0.01)$. However, neither HbA1C levels nor FRS were associated with any changes in LPAs rates (Table 2). Effects of a glycemic burden or elevated $\mathrm{CV}$ risk scores on the percentage of cells positive for PAC-1and P-selectin expression were not found (data not shown).

\section{Discussion}

In this study, we compared blood platelet functionality profiles of high $\mathrm{CV}$ risk diabetic patients, without prior $\mathrm{CV}$ events, to those of matched controls. We found that diabetic patients and matched controls display comparable levels of platelet markers of activation, turnover and leukocyte-platelet interactions.

In the multivariate analysis, an association was found between elevated fasting plasma glucose levels, albeit not HbA1C values or FRS, with an increase of LPAs rates. No association was found between the other activation and aggregation markers with fasting blood glucose, HbA1C 


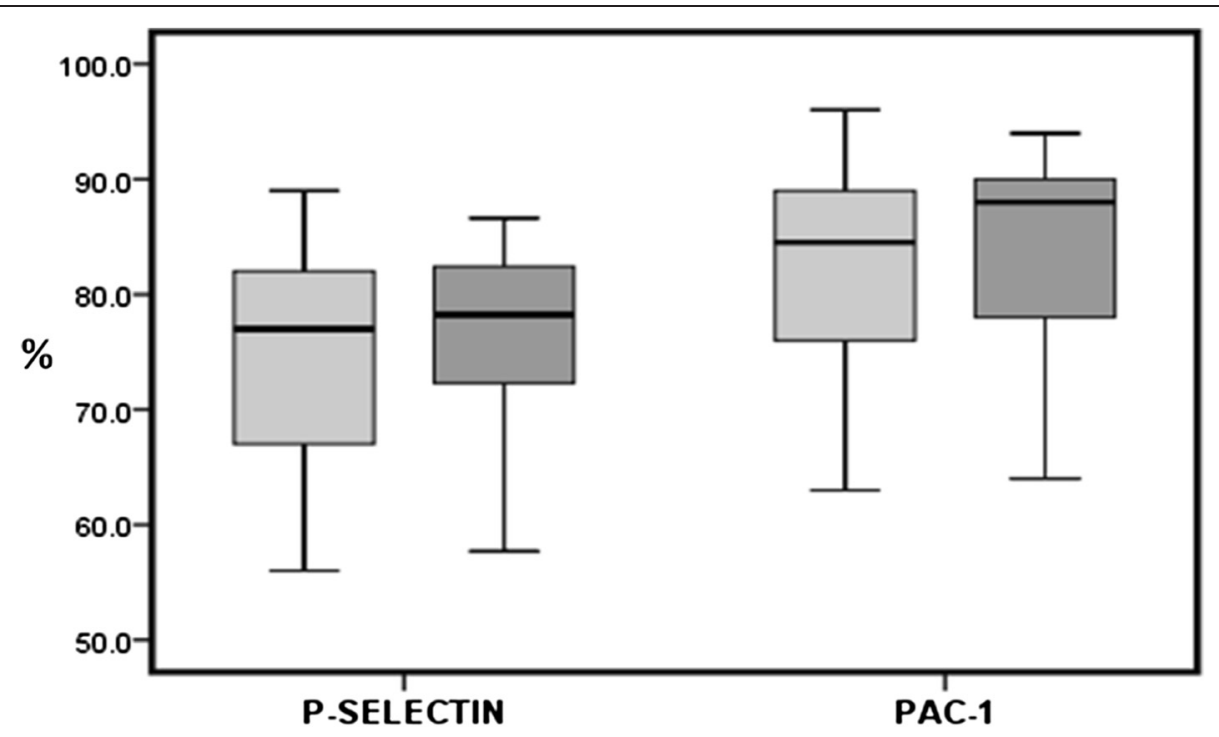

Fig. 2 Percentage of cells positive for PAC-1and P-selectin expression following ADP activation. DM patients are represented in dark gray (right box) and non-diabetic matched controls are represented in light gray (left box). *Abbreviations: DM type 2 diabetes mellitus

levels or FRS. Currently, data regarding the importance of a glycemic burden on platelets activity are conflicting. There are several studies, which demonstrated the importance of intensive glycemic control [34-36] while others have found no effect of intensive glucose control on platelets activity [37].

Several studies have previously demonstrated that platelets taken from diabetic patients show aberrant platelets functionality profiles. However, in most of these trials, platelet hyper-reactivity was found predominantly in patients with either a very high glycemic burden [7, 37,38 ] or documented clinically significant vasculopathy
$[4,37,39-42]$, or to a lesser extent in diabetic patients without angiopathy [34-36, 40, 42, 43]. Conversely, our study population uniquely comprised high CV risk wellcontrolled diabetic patients without prior ischemic events untreated with any anti-platelet medication. Although a few studies have included patients with similar characteristics, they differ significantly from our analysis in several aspects such as primarily focusing on either aspirin resistance among diabetic patients [43] or assessing platelet morphological characteristics in prediabetic patients [35]. In addition, these studies assessed platelets functionality by either the impedance method

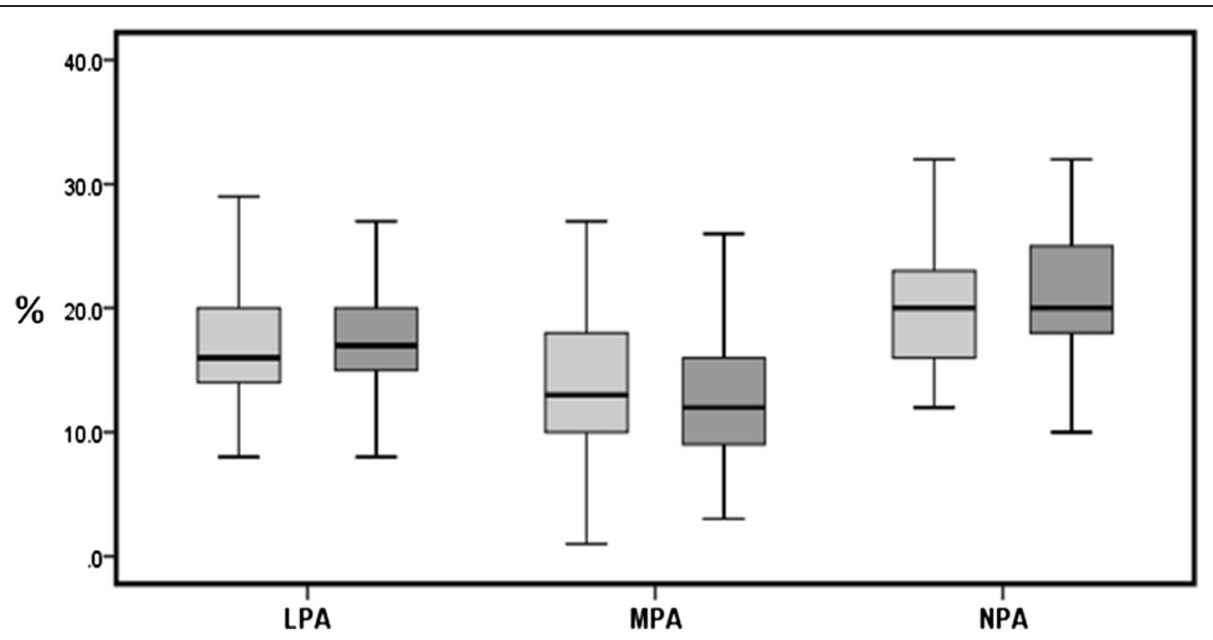

Fig. 3 Percentage of LPAs, MPAs and NPAs following ADP activation. DM patients are represented in dark gray (right box) and non-diabetic matched controls are represented in light gray (left box). *Abbreviations: LPAs Leukocyte-platelet aggregates, MPA Monocyte-platelet aggregates, NPAs Neutrophil-platelet aggregated, DM type 2 diabetes mellitus 
Table 2 Results of multiple linear regression analysis (Dependent variable: percentage of LPAs increase)

\begin{tabular}{llcl}
\hline Covariates & B Coefficient (SE) & Partial correlation & $P$ \\
\hline Age & $-0.09(0.07)$ & -0.15 & 0.235 \\
Gender & $-0.24(1.3)$ & -0.02 & 0.854 \\
HbA1C & $-0.45(0.49)$ & -0.10 & 0.361 \\
FPG & $0.04(0.01)$ & 0.28 & 0.013 \\
LDL & $0.01(0.04)$ & 0.04 & 0.852 \\
HDL & $0.08(0.04)$ & 0.25 & 0.082 \\
FRS & $0.02(0.05)$ & 0.06 & 0.654 \\
\hline
\end{tabular}

LPAs Leukocytes-platelets aggregates, PAC-1 Procaspase activating compound, HbA1c Glycated hemoglobin, FPG Fasting plasma glucose, LDL Low density lipoprotein, HDL High density lipoprotein, eGFR Estimated glomerular filtration rate, FRS Framingham Risk Score, HbA1C Hemoglobin A1C, FPG Fasting Plasma Glucose

through whole-blood aggregometry [34], which might be confounded by various blood components or by light transmission aggregometry (LTA) using platelet rich plasma [43], which is a good assay for evaluation of antiplatelets drug response or bleeding tendency, but not for assessing hyperaggregation.

In our study, however, we assessed platelet activity markers such as P-selectin and PAC-1, as well as leukocyte-platelet aggregates. We tested both activation markers before and after adding ADP. We then evaluated both baseline activation level and the ADP-induced activation response.

Low-dose aspirin remains the cornerstone of antiplatelet therapy for secondary prevention of coronary artery disease, cerebrovascular disease and mortality in diabetic and non-diabetic patients with established vascular disease. However, although its role in the primary prevention of CV events is controversial, many physicians prescribe prophylactic aspirin therapy for their high $\mathrm{CV}$ risk patients, specifically diabetic patients.

During the last 10 years, several large scale prospective randomized controlled trials have been conducted, assessing the beneficiary effect of aspirin as a primary prevention measure among high risk diabetic patients. The Early Treatment Diabetic Retinopathy Study (EDTR) revealed a significant decrease in the relative risk of myocardial infarction at 5 years in patients with type 1 and type $2 \mathrm{DM}$, without any benefit in mortality rates [21]. The Prevention of Progression of Arterial Disease and Diabetes (POPADAD) did not indicate any benefit of aspirin or antioxidants in the primary prevention of $\mathrm{CV}$ events [22]. In the Japanese Primary Prevention of Atherosclerosis with Aspirin for Diabetes (JPAD), primary prevention with aspirin did not reduce the rates of all $\mathrm{CV}$ events, yet the rates of fatal coronary and cerebrovascular events, a secondary end point, were reduced [23]. Furthermore, the recently published data of the Japanese Primary Prevention Project (JPPP) demonstrated that primary prevention with aspirin does not reduce the risk of CV morbidity and mortality in high CV risk patients, including those with DM [24].

In addition, several other trials in which diabetic patients constituted only subgroups within broader trials of aspirin prophylaxis, yielded conflicting results [44-49]. Furthermore meta-analyses demonstrated that primary prevention with aspirin produces only a modest beneficiary effect on CV risk reduction [25-29]. Currently, both ADA guidelines and ESC/EASD guidelines recommend (level C) prophylactic aspirin therapy for high CV risk (10-year risk $>10 \%$ ) diabetic patients [30, 31], which includes most men aged $>50$ years or women aged $>60$ years who have at least one additional major risk factor (family history of $\mathrm{CV}$ disease, hypertension, smoking, dyslipidemia, or albuminuria) [30]. Thus, these recommendations essentially support the use of aspirin as a primary prophylaxis for the majority of diabetic patients.

The results of our study do not support these recommendations, as our multivariate analysis did not detect an association between elevated Framingham Risk Scores and platelet markers of reactivity. Thus, herein, we present novel data suggesting that well-controlled diabetic patients without prior ischemic events have normal platelet functionality profiles, regardless of their CV risk.

The main limitation of our study is that CV risk factors assessed in our population were well-controlled. The enrolled diabetic patients had a low glycemic burden reflected by fasting blood glucose and HbA1C. They also showed minimal aberrancies in lipid metabolism with relatively low smoking rates. Nevertheless, although somewhat limited to a well-controlled diabetic patient population, our results have merit. Diabetic patients whose CV risk factors are well-controlled constitute a large proportion of the diabetic population, with a majority still being widely prescribed unnecessary and potentially hazardous medications such as aspirin [50].

\section{Conclusions}

In conclusion, we demonstrated that in well-controlled high CV risk diabetic patients without prior angiopathy and no anti-aggregate treatment, platelet functionality profiles are normal and comparable to matched controls. Furthermore, for the most part, glycemic burden and $\mathrm{CV}$ risk were not found associated with increased expression of platelet markers of hyper-reactivity. Our findings and recent results of large scale clinical trials collectively undermine the role granted for aspirin as a primary prevention measure in high $\mathrm{CV}$ risk diabetes patients.

\section{Competing interests}

All authors of this manuscript declare that they have no financial or other relationships that might lead to competing interests. 


\section{Authors' contributions}

GS - conceived of the study, participated in its design and coordination, participated in patient enrollment, carried out platelet functionality assays and drafted the manuscript. THA - participated in the study design and helped with patient enrollment, conducted the platelet functionality assays and helped draft the manuscript. YG - participated in the design of the study and helped with patient enrollment and drafting of the manuscript. TS - performed the statistical analysis and helped draft the manuscript. $\mathrm{HH}$ - participated in the study design and performed platelet functionality assays. NR - participated in the design and coordination of the study, performed platelet functionality assays and helped draft the manuscript EG - conceived the study, participated in its design and coordination, drafted the manuscript. The final manuscript has been reviewed and approved by all authors, and all have taken care to ensure the integrity of their work and their scientific reputation.

\section{Acknowledgments}

Dr. Shlomai was supported by The Dr. Pinchas Borenstein Talpiot Medical Leadership Program 2013.

This research was performed as part of the M.D thesis of Ms. Haran-Appel.

\section{Author details}

${ }^{1}$ Department of Internal Medicine D and Hypertension Unit, The Chaim Sheba Medical Center, Derech Sheba 1, Tel Hashomer, Ramat-Gan 52621, Israel. ${ }^{2}$ The Dr. Pinchas Borenstein Talpiot Medical Leadership Program 2013, Tel-Aviv, Israel. ${ }^{3}$ Sackler Faculty of Medicine, Tel-Aviv University, Tel-Aviv, Israel. ${ }^{4}$ Department of Oncology, Chaim Sheba Medical Center, Tel Hashomer, Israel. ${ }^{5}$ Institute of Thrombosis and Hemostasis, Chaim Sheba Medical Center, Tel Hashomer, Israel.

Received: 21 April 2015 Accepted: 6 June 2015

Published online: 12 June 2015

\section{References}

1. Luscher TF, Creager MA, Beckman JA, Cosentino F. Diabetes and vascular disease: pathophysiology, clinical consequences, and medical therapy: Part II. Circulation. 2003:108(13):1655-61. doi:10.1161/01.cir.0000089189.70578.e2.

2. Davi G, Patrono C. Platelet activation and atherothrombosis. N Engl J Med. 2007;357(24):2482-94. doi:10.1056/NEJMra071014.

3. Fuster V, Moreno PR, Fayad ZA, Corti R, Badimon JJ. Atherothrombosis and high-risk plaque: part I: evolving concepts. J Am Coll Cardiol. 2005;46(6):937-54. doi:10.1016/j.jacc.2005.03.074.

4. Kakouros N, Rade JJ, Kourliouros A, Resar JR. Platelet function in patients with diabetes mellitus: from a theoretical to a practical perspective. Int J Endocrinol. 2011;2011:742719. doi:10.1155/2011/742719.

5. Assert R, Scherk G, Bumbure A, Pirags V, Schatz H, Pfeiffer AF. Regulation of protein kinase $C$ by short term hyperglycaemia in human platelets in vivo and in vitro. Diabetologia. 2001:44(2):188-95. doi:10.1007/s001250051598.

6. Ferroni P, Basili S, Falco A, Davi G. Platelet activation in type 2 diabetes mellitus. J Thromb Haemost. 2004;2(8):1282-91. doi:10.1111/j.15387836.2004.00836.x

7. Keating FK, Sobel BE, Schneider DJ. Effects of increased concentrations of glucose on platelet reactivity in healthy subjects and in patients with and without diabetes mellitus. Am J Cardiol. 2003;92(11):1362-5

8. Rollini F, Franchi F, Muniz-Lozano A, Angiolillo DJ. Platelet function profiles in patients with diabetes mellitus. J Cardiovasc Transl Res. 2013;6(3):329-45. doi:10.1007/s12265-013-9449-0.

9. Watala C, Golanski J, Boncler MA, Pietrucha T, Gwozdzinski K. Membrane lipid fluidity of blood platelets: a common denominator that underlies the opposing actions of various agents that affect platelet activation in whole blood. Platelets. 1998;9(5):315-27. doi:10.1080/09537109876564.

10. Winocour PD, Watala C, Perry DW, Kinlough-Rathbone RL. Decreased platelet membrane fluidity due to glycation or acetylation of membrane proteins. Thromb Haemost. 1992:68(5):577-82.

11. Ferretti G, Rabini RA, Bacchetti T, Vignini A, Salvolini E, Ravaglia F, et al. Glycated low density lipoproteins modify platelet properties: a compositional and functional study. J Clin Endocrinol Metab. 2002:87(5):2180-4. doi:10.1210/jcem.87.5.8466.

12. Kobayashi K, Watanabe J, Umeda F, Nawata H. Glycation accelerates the oxidation of low density lipoprotein by copper ions. Endocr J. 1995;42(4):461-5
13. Schmidt AM, Yan SD, Wautier JL, Stern D. Activation of receptor for advanced glycation end products: a mechanism for chronic vascular dysfunction in diabetic vasculopathy and atherosclerosis. Circ Res. 1999:84(5):489-97.

14. Falcon C, Pfliegler G, Deckmyn H, Vermylen J. The platelet insulin receptor: detection, partial characterization, and search for a function. Biochem Biophys Res Commun. 1988;157(3):1190-6.

15. Trovati M, Anfossi G, Massucco P, Mattiello L, Costamagna C, Piretto V, et al. Insulin stimulates nitric oxide synthesis in human platelets, and through nitric oxide, increases platelet concentrations of both guanosine- $3^{\prime}, 5^{\prime}$-cyclic monophosphate and adenosine-3', 5'-cyclic monophosphate. Diabetes. 1997;46(5):742-9.

16. Vaidyula VR, Boden G, Rao AK. Platelet and monocyte activation by hyperglycemia and hyperinsulinemia in healthy subjects. Platelets. 2006:17(8):577-85. doi:10.1080/09537100600760814.

17. Freedman JE. Oxidative stress and platelets. Arterioscler Thromb Vasc Biol. 2008:28(3):s11-6. doi:10.1161/atvbaha.107.159178.

18. Seghieri $G$, Di Simplicio P, Anichini R, Alviggi $L$, De Bellis A, Bennardini $F$, et al. Platelet antioxidant enzymes in insulin-dependent diabetes mellitus. Clin Chim Acta. 2001;309(1):19-23.

19. Patrono C, Davi G. Antiplatelet agents in the prevention of diabetic vascular complications. Diabetes Metab Rev. 1993;9(3):177-88.

20. Levine GN, Bates ER, Blankenship JC, Bailey SR, Bittl JA, Cercek B, et al. 2011 ACCF/AHA/SCAI Guideline for percutaneous coronary intervention. A report of the American college of cardiology foundation/American heart association task force on practice guidelines and the society for cardiovascular angiography and interventions. J Am Coll Cardiol. 2011;58(24):e44-122. doi:10.1016/j.jacc.2011.08.007.

21. ETDRS Investigators. Aspirin effects on mortality and morbidity in patients with diabetes mellitus. Early treatment diabetic retinopathy study report 14. JAMA. 1992;268(10):1292-300.

22. Belch J, MacCuish A, Campbell I, Cobbe S, Taylor R, Prescott R, et al. The prevention of progression of arterial disease and diabetes (POPADAD) trial: factorial randomised placebo controlled trial of aspirin and antioxidants in patients with diabetes and asymptomatic peripheral arterial disease. BMJ (Clinical research ed). 2008:337:a1840. doi:10.1136/bmj.a1840.

23. Ogawa H, Nakayama M, Morimoto T, Uemura S, Kanauchi M, Doi N, et al. Low-dose aspirin for primary prevention of atherosclerotic events in patients with type 2 diabetes: a randomized controlled trial. JAMA 2008;300(18):2134-41. doi:10.1001/jama.2008.623.

24. Ikeda Y, Shimada K, Teramoto T, Uchiyama S, Yamazaki T, Oikawa S, et al. Low-dose aspirin for primary prevention of cardiovascular events in Japanese patients 60 years or older with atherosclerotic risk factors: a randomized clinical trial. JAMA. 2014;312(23):2510-20. doi:10.1001/jama.2014.15690.

25. Pignone M, Alberts MJ, Colwell JA, Cushman M, Inzucchi SE, Mukherjee D, et al. Aspirin for primary prevention of cardiovascular events in people with diabetes: a position statement of the American Diabetes Association, a scientific statement of the American Heart Association, and an expert consensus document of the American College of Cardiology Foundation. Diabetes Care. 2010;33(6):1395-402. doi:10.2337/dc10-0555.

26. Baigent C, Blackwell L, Collins R, Emberson J, Godwin J, Peto R, et al. Aspirin in the primary and secondary prevention of vascular disease: collaborative meta-analysis of individual participant data from randomised trials. Lancet. 2009:373(9678):1849-60. doi:10.1016/s0140-6736(09)60503-1.

27. De Berardis G, Sacco M, Strippoli GF, Pellegrini F, Graziano G, Tognoni G, et al. Aspirin for primary prevention of cardiovascular events in people with diabetes: meta-analysis of randomised controlled trials. BMJ (Clinical research ed). 2009;339:b4531. doi:10.1136/bmj.b4531.

28. Zhang C, Sun A, Zhang P, Wu C, Zhang S, Fu M, et al. Aspirin for primary prevention of cardiovascular events in patients with diabetes: A meta-analysis. Diabetes Res Clin Pract. 2010;87(2):211-8. doi:10.1016/j.diabres.2009.09.029.

29. Calvin AD, Aggarwal NR, Murad MH, Shi Q, Elamin MB, Geske JB, et al. Aspirin for the primary prevention of cardiovascular events: a systematic review and meta-analysis comparing patients with and without diabetes. Diabetes Care. 2009:32(12):2300-6. doi:10.2337/dc09-1297.

30. American Diabetes Association. Standards of medical care in diabetes-2014. Diabetes Care. 2014;37 Suppl 1:S14-80. doi:10.2337/dc14-S014.

31. Ryden L, Grant PJ, Anker SD, Berne C, Cosentino F, Danchin N, et al. ESC guidelines on diabetes, pre-diabetes, and cardiovascular diseases developed in collaboration with the EASD - summary. Diab Vasc Dis Res. 2014:11(3):133-73. doi:10.1177/1479164114525548. 
32. D'Agostino Sr RB, Vasan RS, Pencina MJ, Wolf PA, Cobain M, Massaro JM, et al. General cardiovascular risk profile for use in primary care: the Framingham Heart Study. Circulation. 2008;117(6):743-53. doi:10.1161/circulationaha.107.699579.

33. Nagasawa A, Matsuno K, Tamura S, Hayasaka K, Shimizu C, Moriyama T. The basis examination of leukocyte-platelet aggregates with CD45 gating as a novel platelet activation marker. Int J Lab Hematol. 2013;35(5):534-41. doi:10.1111/ijlh.12051.

34. Harding SA, Din JN, Sarma J, Jessop A, Weatherall M, Fox KA, et al. Flow cytometric analysis of circulating platelet-monocyte aggregates in whole blood: methodological considerations. Thromb Haemost. 2007;98(2):451-6.

35. Shimodaira M, Niwa T, Nakajima K, Kobayashi M, Hanyu N, Nakayama T. Correlation between mean platelet volume and fasting plasma glucose levels in prediabetic and normoglycemic individuals. Cardiovasc Diabetol. 2013;12:14. doi:10.1186/1475-2840-12-14.

36. Stratmann B, Xu T, Meisinger C, Menart B, Roden M, Herder C, et al. PLA1A2 platelet polymorphism predicts mortality in prediabetic subjects of the population based KORA S4-Cohort. Cardiovasc Diabetol. 2014;13:90. doi:10.1186/1475-2840-13-90.

37. Singer J, Weissler Snir A, Leshem-Lev D, Rigler M, Kornowski R, Lev El. Effect of intensive glycemic control on platelet reactivity in patients with long-standing uncontrolled diabetes. Thromb Res. 2014;134(1):121-4. doi:10.1016/j.thromres.2014.05.010.

38. Kimura Y, Takano K, Satoh K, Aida K, Kobayashi T, Ozaki Y. Aspirin half maximal inhibitory concentration value on platelet cyclooxygenase 1 in severe type-2 diabetes mellitus is not significantly different from that of healthy individuals. Clin Appl Thromb Hemost. 2014;20(6):629-36. doi:10.1177/1076029613488934

39. Kabbani SS, Watkins MW, Ashikaga T, Terrien EF, Holoch PA, Sobel BE, et al. Platelet reactivity characterized prospectively: a determinant of outcome 90 days after percutaneous coronary intervention. Circulation. 2001;104(2):181-6.

40. Tschoepe D, Roesen P, Esser J, Schwippert B, Nieuwenhuis HK, Kehrel B, et al. Large platelets circulate in an activated state in diabetes mellitus. Semin Thromb Hemost. 1991;17(4):433-8. doi:10.1055/s-2007-1002650.

41. Yngen M, Norhammar A, Hjemdahl P, Wallen NH. Effects of improved metabolic control on platelet reactivity in patients with type 2 diabetes mellitus following coronary angioplasty. Diab Vasc Dis Res. 2006;3(1):52-6. doi:10.3132/dvdr.2006.008.

42. Lowe LP, Greenland P, Ruth KJ, Dyer AR, Stamler R, Stamler J. Impact of major cardiovascular disease risk factors, particularly in combination, on 22-year mortality in women and men. Arch Intern Med. 1998;158(18):2007-14.

43. Lemkes BA, Bahler L, Kamphuisen PW, Stroobants AK, Van Den Dool EJ, Hoekstra JB, et al. The influence of aspirin dose and glycemic control on platelet inhibition in patients with type 2 diabetes mellitus. J Thromb Haemost. 2012;10(4):639-46. doi:10.1111/j.1538-7836.2012.04632.x.

44. Peto R, Gray R, Collins R, Wheatley K, Hennekens C, Jamrozik K, et al. Randomised trial of prophylactic daily aspirin in British male doctors. Br Med J (Clin Res Ed). 1988;296(6618):313-6.

45. Steering Committee of the Physicians' Health Study Research Group. Final report on the aspirin component of the ongoing Physicians' Health Study. N Engl J Med. 1989;321(3):129-35. doi:10.1056/nejm198907203210301.

46. The Medical Research Council's General Practice Research Framework. Thrombosis prevention trial: randomised trial of low-intensity oral anticoagulation with warfarin and low-dose aspirin in the primary prevention of ischaemic heart disease in men at increased risk. Lancet. 1998;351(9098):233-41.

47. Hansson L, Zanchetti A, Carruthers SG, Dahlof B, Elmfeldt D, Julius S, et al. Effects of intensive blood-pressure lowering and low-dose aspirin in patients with hypertension: principal results of the Hypertension Optimal Treatment (HOT) randomised trial. HOT Study Group. Lancet. 1998;351(9118):1755-62.

48. Sacco M, Pellegrini F, Roncaglioni MC, Avanzini F, Tognoni G, Nicolucci A. Primary prevention of cardiovascular events with low-dose aspirin and vitamin E in type 2 diabetic patients: results of the Primary Prevention Project (PPP) trial. Diabetes Care. 2003;26(12):3264-72.

49. de Gaetano G. Low-dose aspirin and vitamin E in people at cardiovascular risk: a randomised trial in general practice. Collaborative Group of the Primary Prevention Project. Lancet. 2001;357(9250):89-95.

50. MacDonald TM, Morant SV, Robinson GC, Shield MJ, McGilchrist MM, Murray FE, et al. Association of upper gastrointestinal toxicity of non-steroidal antiinflammatory drugs with continued exposure: cohort study. BMJ (Clinical research ed). 1997;315(7119):1333-7.

\section{Submit your next manuscript to BioMed Central and take full advantage of:}

- Convenient online submission

- Thorough peer review

- No space constraints or color figure charges

- Immediate publication on acceptance

- Inclusion in PubMed, CAS, Scopus and Google Scholar

- Research which is freely available for redistribution

Submit your manuscript at www.biomedcentral.com/submit 